\title{
Student Reflective Practices
}

\author{
Herbert Rau \\ Utica College, Utica, USA
}

\begin{abstract}
For decades, reflective practices for professionals and students have been recommended by scholars. It is not clear, however, whether significant attention has been focused to understand if an inherent tendency towards the practice of reflection is demonstrated by students. A statistical study was conducted to determine whether students demonstrate a propensity towards reflection, and if a tendency is identified, how that strategy is expressed. Further, utilizing the Human Dynamics model of Seagal and Horne, an attempt is made to determine whether particular dynamics exploit different reflective strategies. The study suggests that not all students reflect on their learnings, and for those who do reflect, the purpose of their reflections varies from individual to individual. The results of the study also suggest that providing a clear structure for reflection will help students be successful with this discipline.
\end{abstract}

Keywords: reflective practices, human dynamics, learning organization, personal mastery

\section{Introduction}

D'Aveni introduced the concept of "hyper-competition" to describe the tumultuous environment in which many organizations operate (D’Aveni, 1994). According to D'Aveni, the hyper-competitive environment is a factor of many competent and aggressive global organizations competing in four arenas; price and quality, timing and know-how, protection and invasion of markets, and the availability of capital and alliances. The result of this competitive environment is a rapidly changing environment where today's competitive advantage is no longer a certainty for tomorrow, and complexity rules the day.

This challenging environment is difficult to manage. Obviously, part of the difficulty is due to the skill, knowledge, abilities, and execution of strategy by the competition. However, Sterman (2000) notes a different type of complexity may be due to the faulting thinking of managers. As Sterman has noted, a number of behaviors and patterns of action that cause problems for managers and their companies are present for most individuals including (but are not limited to) confirmation bias, an early termination of evidence search, inertia, limited experience, wishful thinking, magical thinking, halo effect, memory bias, and group-think.

A potential third factor in managing the complexity and confusion of the contemporary world is the issue of reflective thinking performed by managers, and whether individuals and organization are utilizing this practice. Drucker wrote about the need to reflect (Drucker, 1967), and Schon discusses the importance of being a “reflective practitioner”, and introduced the concepts of "reflection-in-action”, and "reflection-on-action” (Schon, 1983). However, it is not clear that a significant amount of attention has been devoted to understanding whether

Herbert Rau, Ph.D., Department of Business and Economics, Utica College.

Correspondence concerning this article should be addressed to Herbert Rau, 2 Hemlock Hill Road, Cape Elizabeth, Maine 04107, USA.E-mail: hrau@utica.edu. 
individuals have natural tendencies towards reflection, let alone whether it is reflection-in-action, and reflection-on-action. Along these lines, Pyc and Rawson (2010) have noted that self-testing improves memory and learning, and reflective practices may help to contribute to this improvement. It is the position of this paper that reflection is an important practice, and students should be encouraged to integrate this practice in their daily lives. This perspective is shared by others (McDrury \& Alterio, 2002; Boud, Cohen, \& Walker, 1993; King \& Kitchner, 1981; Magolda, 2001).

Gibbs has contributed to the process of reflective thinking by including the aspect of evaluation and analysis including the element of emotions associated with a particular event (Gibbs, 1988). While this is important, considering the work of Seagal and Horne (1997), this may have limitations due to how individuals perceive and interact with their environment.

The Human Dynamics model developed by Seagal and Horne postulates that there are three fundamental principles as to how people understand their world: the physical, mental and emotional principles. In this regard, the physical principle refers to the tendency for an individual to "actualize" something. The emotional principle addresses the relational aspects of the individual with the world, that is, how does the individual relate to other people, ideas, and physical reality. The mental principle addresses objectivity, vision, and purpose.

However, these principles do not stand in isolation. Each human has these three principles in their persona. According to the Human Dynamic model, one principle will be dominant, while the other principles will be of secondary and tertiary influence. Since the Human Dynamic model recognizes that each person possesses all three principles, when two principles are integrated to describe an individual, it forms a unit called a "dynamic".

According to the Human Dynamics model, there are nine potential dynamics: three for individuals who are mentally centered, three for individuals who are emotionally centered, and three for individuals who are physically centered. The first dominant principle addresses how the individual processes their world. The second principle addresses what is being processed, and the third principle addresses why. Therefore, an individual who is emotionally centered, and has as their second principle the mental component would represent a unique dynamic that characterizes how that individual understands and processes their world. This individual would be identified as an "emotional-mental" dynamic (EM), and would be processing their world of ideas from a relational perspective.

The Human Dynamic model recognizes that there is great variety expressed for each dynamic. The model proposes that all more than seven billion people in the world are represented by nine dynamics, yet there is variety within the bounds of the dynamics in how the combined principles are expressed. While there is a great range of "expression” demonstrated for the dynamics, experience with more than 100,000 individuals suggests that five dynamics predominate. The majority of individuals of Asian heritage tend to identify with the attributes of the physically centered dynamic (Physical Mental and Physical Emotional), while individuals of European ancestry tend to resonate with the attributes of the emotionally centered dynamics (Emotional Physical and Emotional Mental). The Mental Physical dynamic tends to be represented mostly be individuals of European origin, yet this dynamic represents fewer than $5 \%$ of individuals who have been observed. The remaining four dynamics are significantly smaller in size. What is important in this context, is that the different dynamics tend to demonstrate particular tendencies for different actions and different preferences in communication. Understanding the different dynamics is helpful in enhancing learning and communication with different individuals. 
Considering the work of Schon and Gibbs, an inquiry was undertaken to determine if post-secondary school students have a natural tendency towards reflection-in-action or reflection-on-action, and whether any tendency for reflective practices was discernable utilizing the Human Dynamics model. In alignment with the work of Argyris regarding the "reflexive loop" and "ladder of inference" (Argyris, 1982), it is believed that an understanding of the reflective practices of students is important in order to help students be more successful with their careers, and to more effectively guide their organizations within the current hyper-competitive environment.

\section{Methodology}

End of class surveys were conducted for two undergraduate strategy classes (one evening and one day class), and for two online graduate MBA core classes. A total of 95 students voluntarily and anonymously participated with the survey. The survey was designed to achieve a variety of purposes including the determination of learning style preferences, perspective on the course that had just been completed, value of the class, and reflection practices. Generally, the specific questions were asked using a nine-point Likert scale. For the questions pertinent to reflective practices, the questions were:

(1) What is your preferred style of learning?

- Reading and studying alone;

- Discussion and debate with classmates in class;

- Group work in class and outside of the class;

- Lecture.

(2) What is your primary orientation for your actions and work?

- Principles;

- Tasks to be accomplished;

- Social relations.

(3) Do you consider yourself to be a self-starter (need little direction to accomplish tasks)?

- Score of " 1 " equals "Not at all”, score of "9" equals "Dramatically".

(4) Do you consider yourself to be creative?

- Score of "1" equals "Not at all";

- Score of "9" equals "Dramatically”.

(5) Do you see yourself as more idea-based, or activity-based (getting things done)?

- Score of "1" equals "Idea";

- Score of "9" equals “Activity”.

(6) How flexible do you think you are?

- Score of "1" equals "Not at all”;

- Score of "9" equals "Dramatically".

(7) How much time do you need to recall facts, situations experiences?

- Score of "1" equals "Instant recall, general information, but few details";

- Score of "9" equals "Sometimes hours, but lot of details".

(8) Do you like working with others in groups?

- Score of "1" equals "Not at all”; 
- Score of "9" equals "Dramatically preferred".

(9) How much spontaneity about learning do you like?

- Score of "1" equals "Detailed idea of what is going to be covered before learning takes place";

- Score of "9" equals "Free flow learning can occur at any time, on any subject, anywhere".

(10) How much structure do you need to be able to learn?

- Score of "1" equals "Lots of structure";

- Score of "9" equals "No structure necessary".

(11) How often do you reflect on your learning? If never skip to question \#14.

- Score of "1" equals "Never";

- Score of "9"equals "All the time".

(12) If you reflect on your learning, do you reflect:

- While learning?

- Immediately after learning?

- At a time somewhat distant from when the learning took place?

(13) If you reflect on your learning, how much do you reflect on:

- What you learned?

Score of "1" equals "Never";

Score of "9" equals "Always".

- How you learned it?

Score of "1" equals "Never";

Score of "9" equals "Always".

- Why you learned it?

Score of "1" equals "Never";

Score of "9" equals "Always".

- How you would use it?

Score of "1" equals "Never";

Score of "9" equals "Always".

- How it integrates into other learning?

Score of "1" equals "Never";

Score of "9" equals "Always".

- How you could learn more easily and more effectively?

Score of "1" equals "Never";

Score of "9"” equals "Always".

The scores from individual surveys were tabulated and analyzed using the JMP and Minitab statistical packages. Distribution, Fit $Y$ by $X$, Fit Model, Partitioning, Matched Pairs, ANOVA, Main Effects, Dendrogram Assessment Clustering, and Contour Plotting analysis were performed across the questions noted above.

Questions \#1 and \#2 were designed to determine whether alignment with the Human Dynamics model was discernable with this group of students. The elements of question \#1 relate to how the preferences of the 100,000 plus individuals who have been observed in Human Dynamic workshops have identified themselves as 
processing the world in a manner consistent with which the five predominant dynamics prefer to learn. The elements of question \#2 were also designed to determine whether alignment with the surveyed students exist with the Human Dynamics model.

\section{Results}

Table 1 provides the gross statistics for the three classes. Figure 1 is the histogram for the question "How often do you reflect on your learning?”. For Figure 2, it is noted that 19 individuals either never reflected or rarely reflected on their learning (scores $0,1,2, \& 3$ ) accounting for $20 \%$ of the sampled students, 30 students reflected frequently on their learning (scores 7, 8, \& 9) accounting for 32\% of the sampled students, and the remaining 46 students (48\% of the population) occasionally reflected on their learning. The majority of undergraduate night students tended to reflect some time distant from when they learned the subject matter (see Table 1), while the majority of undergraduate day students tended to reflect shortly after learning. The graduate students tended to split their reflective style between during learning, and at some distance from when they learned.

It is interesting to note from Table 1, that from an "orientation of action and work" perspective, undergraduate night students were predominantly aligned with social relationships, while the undergraduate day and graduate students were more aligned with "tasks to be accomplished". This alignment by the night students with social relationships is not unexpected with regard to the Human Dynamics model, however, at this time, it is not clear why day undergraduate and graduate students are more focused on "work to be accomplished".

Table 1 also notes the differences in learning styles between the various classes. Graduate students tended to prefer to read and study alone (37.5\%). The undergraduate day class seemed to prefer discussion and debate with classmates, and group work within and external to the class. The undergraduate night students preferred discussion and debate, and lecture.

Table 1

Class Statistics

\begin{tabular}{lccc}
\hline & Undergraduate night (\%) & Undergraduate day (\%) & Graduate (\%) \\
\hline Style & & 3.3 & 37.5 \\
Unidentified & 10 & 4.9 & 20.8 \\
Reading and studying alone & 40 & 52.5 & 20.8 \\
Discussion and debate with classmates in class & 32.8 & 20.8 \\
Group work in class and outside of the class & 20 & 6.5 & 17 \\
Lecture & 30 & 5 & 83 \\
Orientation & & 8 & 75 \\
Unidentified & 20 & 11 & 45 \\
Principles & & & 10 \\
Tasks to be accomplished & 80 & 20 & 45 \\
Social relationships & & 47.5 & \\
Reflection style & 20 & 8.2 & 24.6 \\
Unidentified & 20 & & \\
While learning & & & \\
Shortly after learning & 60 & & \\
Some time later & & & \\
\hline
\end{tabular}




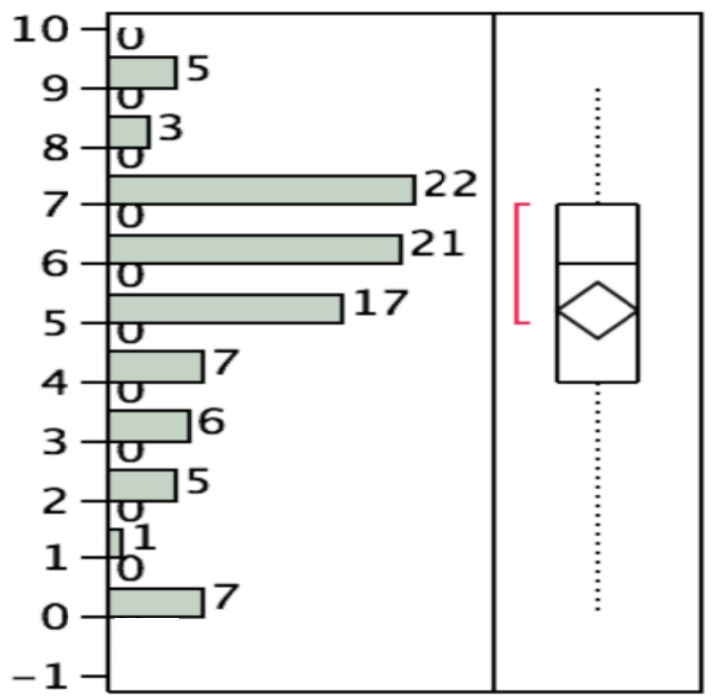

Figure 1. Histogram of how often reflect on learning.
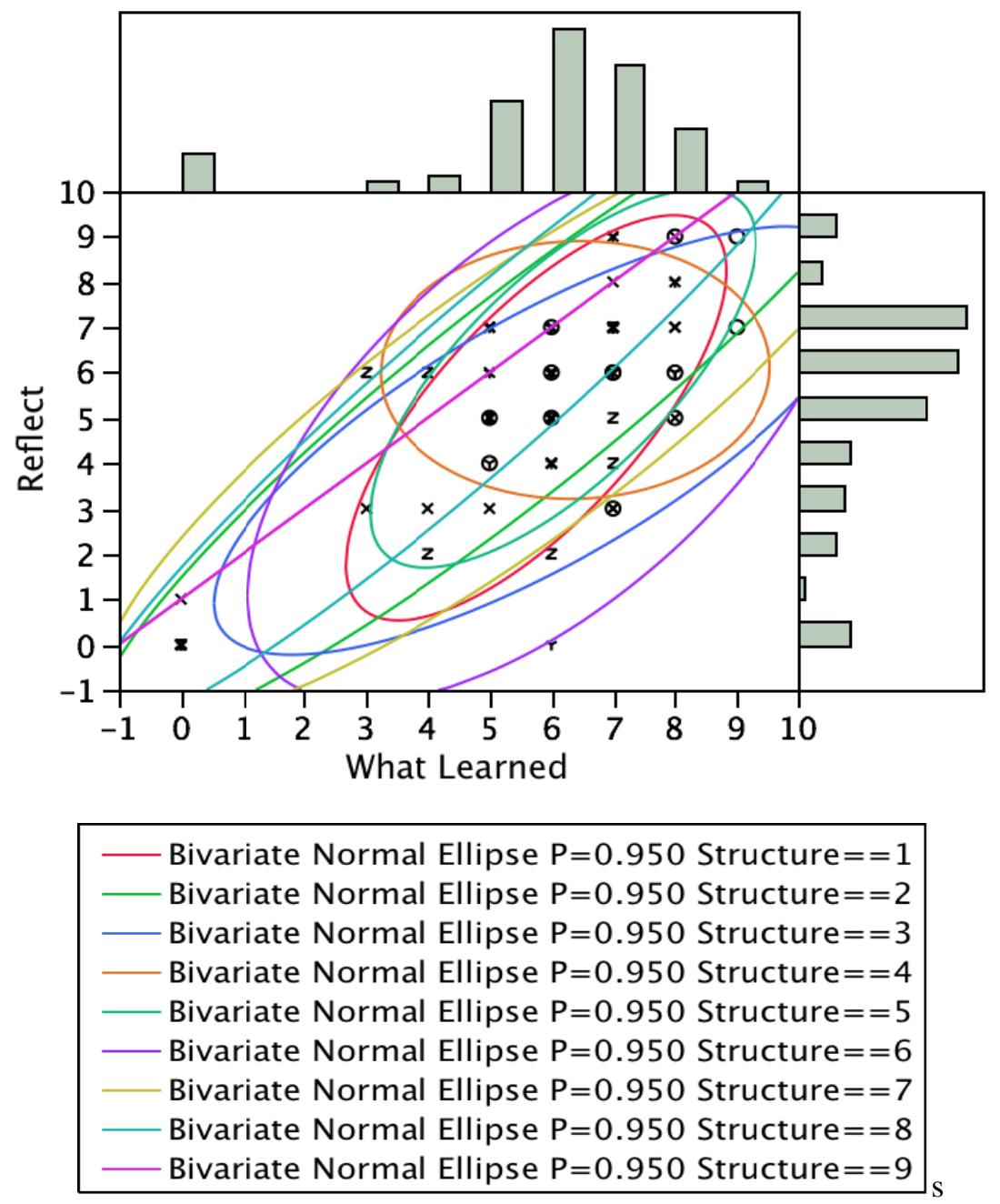

Figure 2. Sample fit $Y$ by $X$ analysis correlation bivariate fit of reflect by what learned. 
Figure 2 and Table 2 are a representative sample of the results for a fit $Y$ by $X$ analysis that was used in the JMP statistical package. A total of 125 simulations were performed, resulting in 950 separate correlation results. Highlights of the results of this correlation work are described below. This analysis was performed to determine whether there were factors that correlated with reflective practices. The correlation studies scanned the full range of responses for each student and each factor (score ranges from 1 to 9). If correlation was consistent across the full spectrum of scores, it was determined that the factor(s) were important to reflection. No factor achieved $100 \%$ correlation, however a number of factors demonstrated general correlation across the score ranges.

Table 2

Sample Fit Y by X Analysis Correlation

\begin{tabular}{|c|c|c|c|c|c|}
\hline Variable & Mean & Std. Dev. & Correlation & Signif. Prob. & Number \\
\hline \multicolumn{6}{|c|}{ Correlation structure 1} \\
\hline What learned & 5.75 & 1.258306 & 0.725476 & 0.2745 & 4 \\
\hline Reflect & 5 & 1.825742 & & & \\
\hline \multicolumn{6}{|c|}{ Correlation structure 2} \\
\hline What learned & 5.333333 & 2.73252 & 0.931793 & 0.0068 & 6 \\
\hline Reflect & 5.333333 & 3.011091 & & & \\
\hline \multicolumn{6}{|c|}{ Correlation structure 3} \\
\hline What learned & 5.833333 & 2.167249 & 0.76045 & 0.0041 & 12 \\
\hline Reflect & 4.5 & 1.930615 & & & \\
\hline \multicolumn{6}{|c|}{ Correlation structure 4} \\
\hline What learned & 6.388889 & 1.289728 & 0.023992 & 0.9247 & 18 \\
\hline Reflect & 6.055556 & 1.161754 & & & \\
\hline \multicolumn{6}{|c|}{ Correlation structure 5} \\
\hline What learned & 6.1875 & 1.276388 & 0.714882 & 0.0019 & 16 \\
\hline Reflect & 5.875 & 1.707825 & & & \\
\hline \multicolumn{6}{|c|}{ Correlation structure 6} \\
\hline What learned & 5.928571 & 1.979288 & 0.617841 & 0.0185 & 14 \\
\hline Reflect & 4.857143 & 2.507133 & & & \\
\hline \multicolumn{6}{|c|}{ Correlation structure 7} \\
\hline What learned & 5.230769 & 2.681848 & 0.865976 & 0.0001 & 13 \\
\hline Reflect & 5 & 2.798809 & & & \\
\hline \multicolumn{6}{|c|}{ Correlation structure 8} \\
\hline What learned & 4.666667 & 2.581989 & 0.954987 & 0.0030 & 6 \\
\hline Reflect & 5.5 & 3.082207 & & & \\
\hline \multicolumn{6}{|c|}{ Correlation structure 9} \\
\hline What learned & 4 & 5.656854 & 1 & & 2 \\
\hline Reflect & 5 & 5.656854 & & & \\
\hline
\end{tabular}

It should be noted for the above table, that $0=2$ and $S=1$ relate to the second orientation element (question \#2 of the survey) and the first style element (question \#1 in the survey). Likewise, $S=2, S=3, S=4$, and $O=1$ reflect their respective elements within questions \#1 and \#2.

Figure 3 is a sample of oneway density analysis that was performed using the JMP statistical package. A total of 21 density plots were analyzed, and were used in conjunction with the least square fit models (a sample result is presented in Figure 4). 
Table 3 and Figure 5 show the statistics for the various factors that were investigated. Figure 6 and Tables 4-5 are a representative sample of the 95 distribution plots that were generated from the JMP statistical package and used in this analysis.
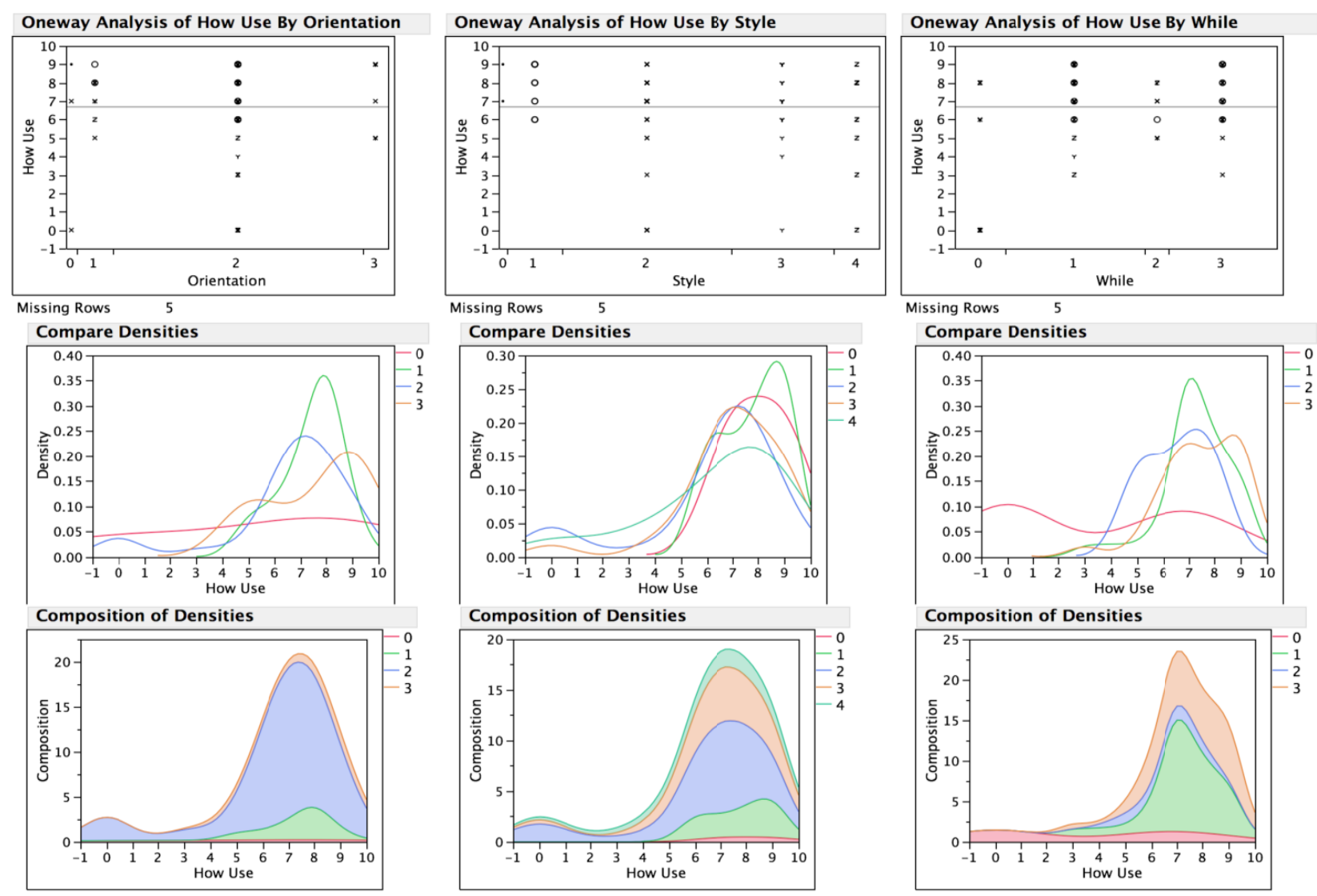

Figure 3. Sample fit $X$ by $Y$ oneway density analysis.

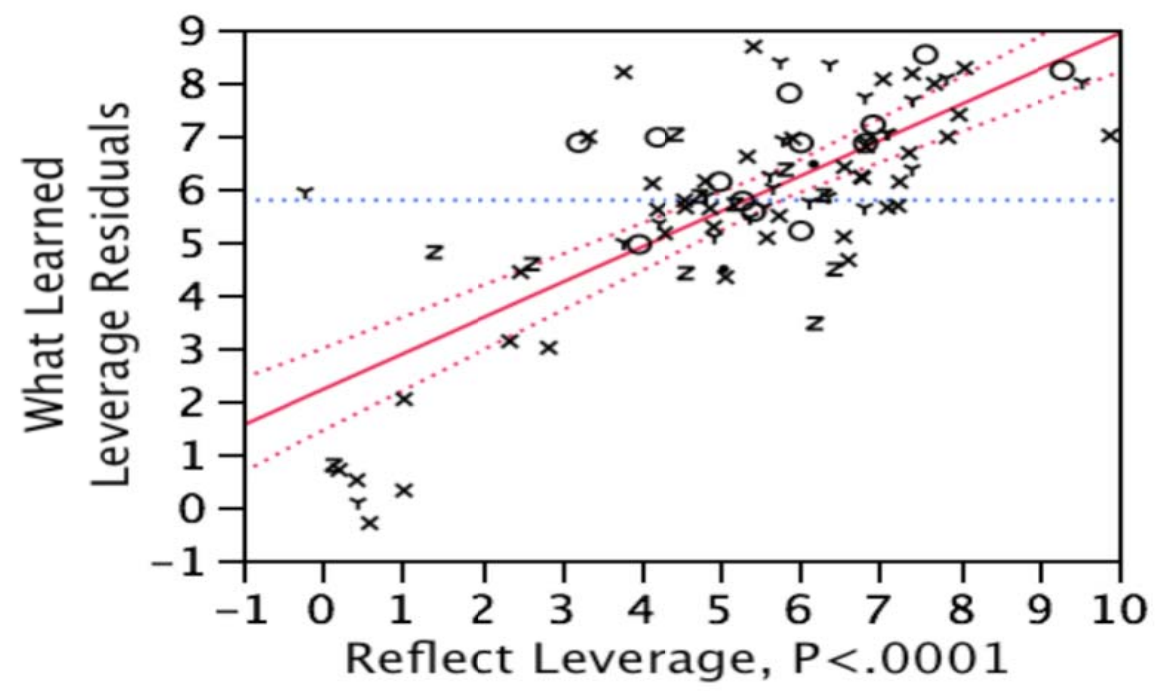

Figure 4. Sample fit model (least squares) what learned by reflect. 
Table 3

\section{Significant Groups Means and Standard Deviations for Investigated Factors}

\begin{tabular}{|c|c|c|c|c|c|c|c|c|c|c|c|c|c|c|c|c|c|}
\hline & & Count & Self & Creative & Activity & Flexible & $\begin{array}{l}\text { Recall } \\
\text { time }\end{array}$ & Groups & Spontaneity & Structure & Reflect & $\begin{array}{l}\text { What } \\
\text { learned }\end{array}$ & $\begin{array}{l}\text { How } \\
\text { learned }\end{array}$ & $\begin{array}{l}\text { Why } \\
\text { learned }\end{array}$ & How use & $\begin{array}{l}\text { How } \\
\text { integrates }\end{array}$ & $\begin{array}{l}\text { Learn } \\
\text { easier }\end{array}$ \\
\hline $\mathrm{O}=2$ & Mean & 11 & 6.55 & 6.18 & 6.09 & 6.64 & 4.73 & 4.91 & 4.91 & 4.18 & 5.73 & 7.0 & 5.55 & 6.55 & 7.64 & 6.91 & 5.82 \\
\hline$S=1$ & Std. dev. & & 1.97 & 2.48 & 1.51 & 1.36 & 1.42 & 1.97 & 2.43 & 1.33 & 1.62 & 1.41 & 1.92 & 1.57 & 1.29 & 1.51 & 1.66 \\
\hline $\mathrm{O}=2$ & Mean & 29 & 6.62 & 7.41 & 6.21 & 6.45 & 4.76 & 5.28 & 5.34 & 4.79 & 4.79 & 5.28 & 4.48 & 5.45 & 6.07 & 5.41 & 5.48 \\
\hline$S=2$ & Std. dev. & & 1.84 & 1.30 & 2.24 & 1.30 & 1.92 & 1.65 & 2.33 & 1.92 & 2.34 & 2.42 & 2.44 & 2.76 & 2.70 & 2.86 & 2.71 \\
\hline $\mathrm{O}=2$ & Mean & 23 & 6.13 & 7.04 & 6.09 & 6.65 & 5.39 & 7.61 & 5.43 & 4.83 & 5.21 & 6.1 & 5.15 & 5.05 & 6.85 & 6.25 & 5.8 \\
\hline$S=3$ & Std. dev. & & 1.66 & 1.85 & 2.19 & 1.27 & 1.88 & 1.34 & 2.45 & 1.95 & 2.49 & 1.68 & 2.13 & 2.65 & 2.06 & 1.83 & 2.24 \\
\hline $\mathrm{O}=2$ & Mean & 10 & 5.2 & 6.6 & 5.6 & 6.9 & 4.6 & 4.9 & 5.7 & 4.9 & 4.44 & 4.78 & 4.33 & 4.89 & 6.11 & 5.44 & 5.56 \\
\hline$S=4$ & Std. dev. & & 1.40 & 0.97 & 2.59 & 1.20 & 1.43 & 2.08 & 1.57 & 2.28 & 2.19 & 2.23 & 2.45 & 2.37 & 2.98 & 3.05 & 2.88 \\
\hline $\mathrm{O}=1$ & Mean & 6 & 6.5 & 7.0 & 6.17 & 6.83 & 4.83 & 6.83 & 6.17 & 4.0 & 6.33 & 6.67 & 5.5 & 6.5 & 7.2 & 6.67 & 6.17 \\
\hline$S=2$ & Std. dev. & & 1.64 & 1.10 & 1.94 & 1.47 & 1.17 & 1.33 & 2.23 & 1.67 & 2.16 & 1.04 & 1.64 & 1.38 & 1.31 & 2.16 & 2.23 \\
\hline
\end{tabular}

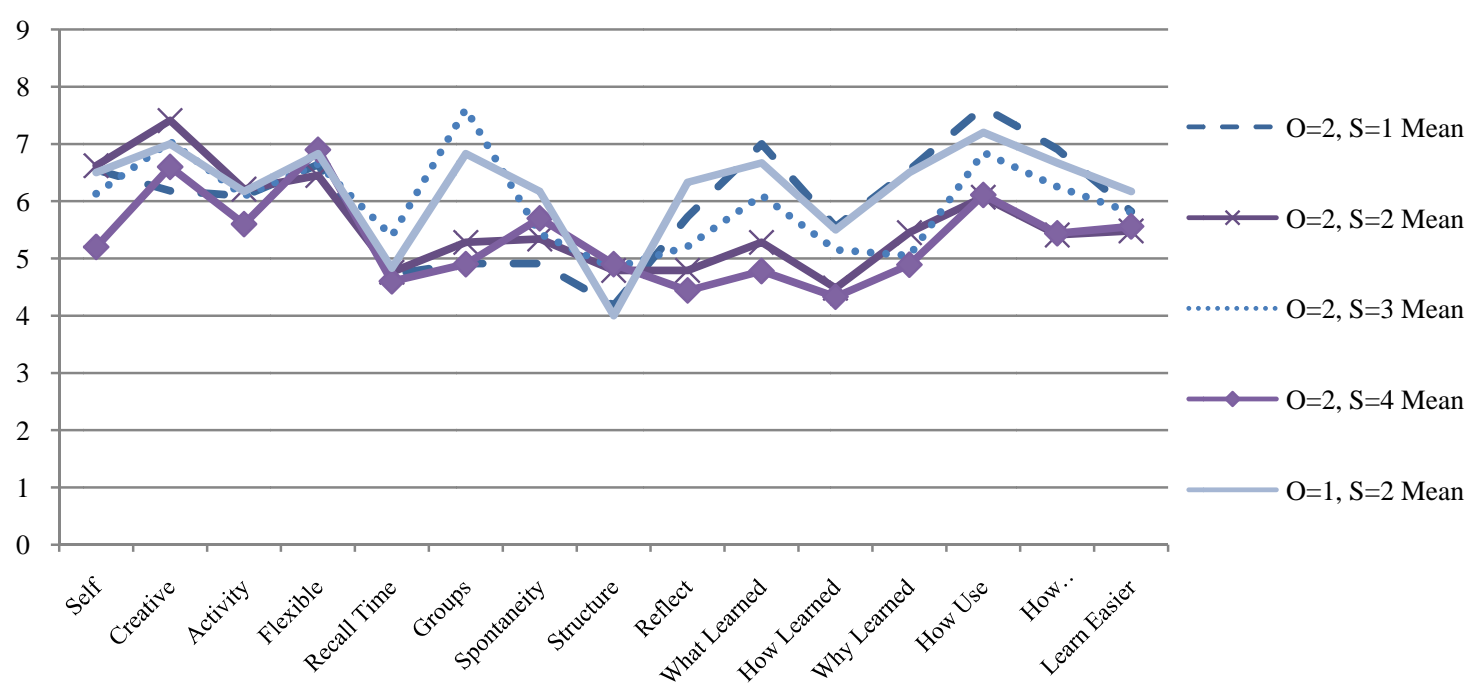

Figure 5. Chart from table of means for investigated factors.

Activity

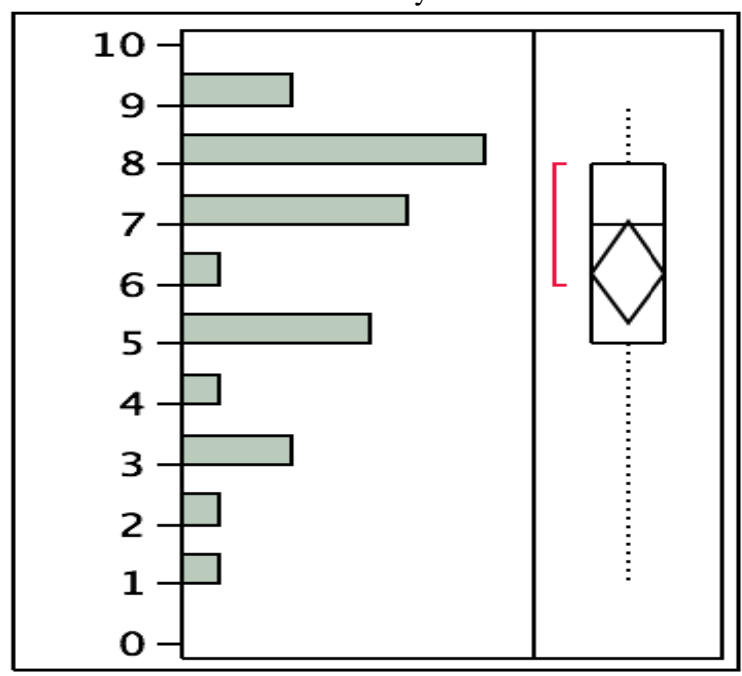

Figure 6. Sample distribution of activity factor generated by orientation and style. 
Table 4

Sample Distribution of Activity Factor Generated by Orientation and Style

\begin{tabular}{cll}
\hline Quantiles & Quartile & Activity \\
\hline $100.0 \%$ & maximum & 9.0000 \\
$99.5 \%$ & & 9.0000 \\
$97.5 \%$ & & 9.0000 \\
$90.0 \%$ & & 9.0000 \\
$75.0 \%$ & quartile & 8.0000 \\
$50.0 \%$ & median & 7.0000 \\
$25.0 \%$ & quartile & 5.0000 \\
$10.0 \%$ & & 3.0000 \\
$2.5 \%$ & & 1.0000 \\
$0.5 \%$ & & 1.0000 \\
$0.0 \%$ & minimum & 1.0000 \\
\hline
\end{tabular}

Table 5

Sample Distribution of Activity Factor Generated by Orientation and Style

\begin{tabular}{lc}
\hline Moments & Activity \\
\hline Mean & 6.2068966 \\
Std. dev. & 2.2421181 \\
Std. err. mean & 0.4163509 \\
Upper 95\% mean & 7.0597527 \\
Lower 95\% mean & 5.3540404 \\
N & 29 \\
\hline
\end{tabular}

$\mathrm{N}$

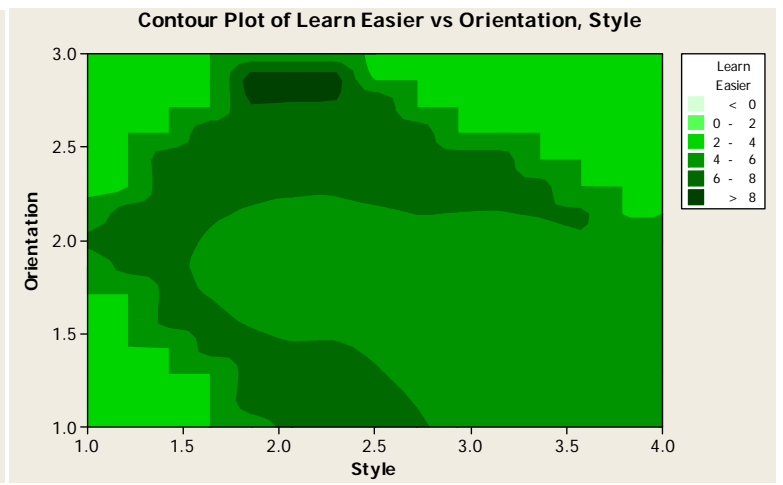

Figure 7. Sample contour plots generated for orientation, style.
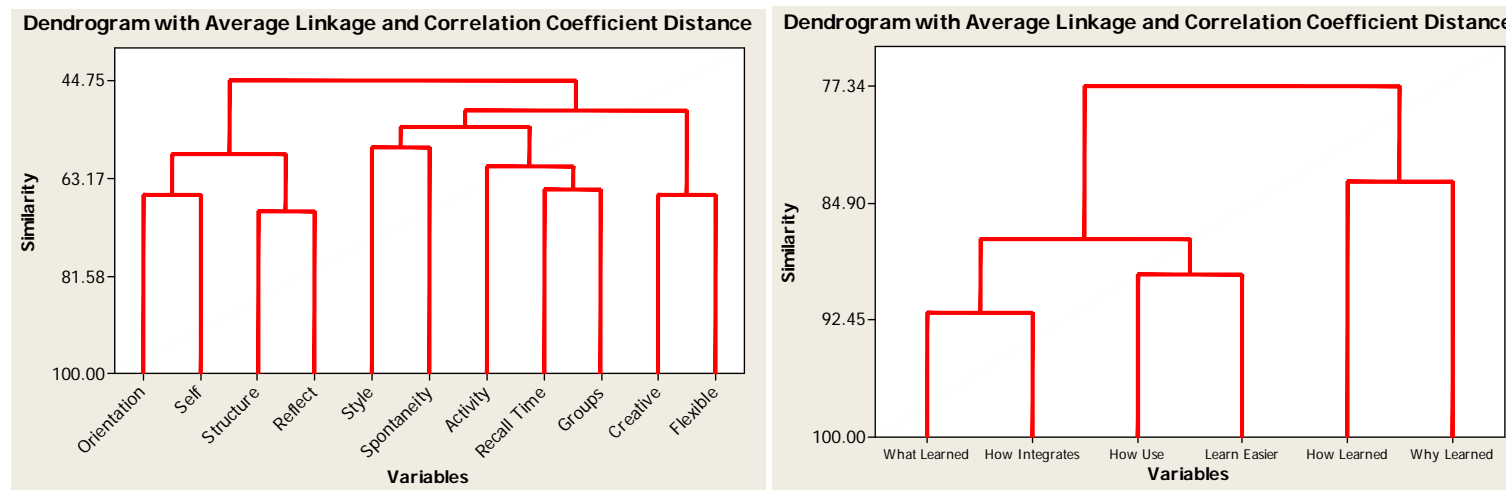

Figure 8. Dendrogram assessment cluster plot. 
Figure 7 and Figure 8 are representative samples of Contour Plot Analysis and Dendrogram Analysis generated through the Minitab statistical package.

\section{Observations}

A number of different observations were made from the fit $Y$ by $X$ analysis for style of learning with particular reflection practices:

- Fifty three percent of the "alone” style of learning reflect some time after their learning, while $38 \%$ reflect during their learning;

- Forty four percent of the “discussion” style of learning reflect during learning, 29\% reflect some time later. Interestingly, 20\% of this style of learning didn’t reflect at all;

- Thirty seven percent of the "group work" style of learning reflect during and some time distant from the learning, and 17\% did not reflect at all;

- Out of 95 students (grad and undergrad), 13\% prefer lecture, 28\% prefer group work, 43\% prefer discussion, $14 \%$ prefer alone, and $2 \%$ didn't identify;

- Globally, 14 students didn’t reflect at all (15\%), 40 students reflect while learning (44\%), 7 reflected at a short time after learning (8\%), and 30 reflected distant from learning (33\%).

Observation made for the fit $Y$ by $X$ analysis for learning includes:

• Undergraduate night students had a strong correlation with "what is learned”, “why it was learned”, "how it was learned”, "how to use it”, "how it integrates with what has been learned elsewhere”, and "how to learn easier";

- Undergraduate day students had a strong correlation with "what was learned”, "how to use it”, but this group had only a moderate correlation with "how it was learned”, "why it was learned”, "how it integrates with other learning”, and "how to learn easier";

- Graduate students show a moderate correlation with "how to use it", but demonstrated no correlation with the other factors.

For "Learning Styles", the observations for the Fit $Y$ by $X$ analysis include:

• "Lecture” learning style demonstrated a strong correlation for being “creative” and "activity”. Only a modest correlation existed for "recall time” and "reflection”, and a negative correlation existed for "flexibility" and "activity";

• "Group work” learning style demonstrated a modest correlation with “activity” and "recall time”, “creative” and "spontaneity”, and "self-starter" and "spontaneity”. A modest negative correlation exists for "recall time” and "structure";

• "Discussion” learning style demonstrated only a modest correlation with "spontaneity" and "structure”;

• “Alone” learning style demonstrated a modest correlation for "flexible” and "groups”, “flexible” and “structure”, “flexible” and “creative”, and a modest negative correlation for "activity” and "reflect”.

Correlation appears to be consistent for the following facilitating and learning factors:

- "Structure" and "what is learned";

- "Structure" and "how it is learned";

• "Structure" and "why it is learned"; 
- "Structure" and "how to use";

- "Structure and "easier to learn";

- "Spontaneity" and "how to use";

- "Spontaneity" and "how it integrates";

- "Groups" and "what is learned";

- "Groups" and "how to use";

• "Recall time" and "what is learned";

- "Recall time" and "why it is learned";

- "Recall time" and "how to use";

- "Flexibility" and "what is learned";

- "Flexibility" and "how it integrates";

- "Activity" and "how to use”;

- "Activity" and "how it integrates";

- "Self-starter" and "what is learned";

- "Self-starter" and "how to use".

Style appears to demonstrate fit $Y$ by $X$ correlation with:

- "Lecture" and "what learned";

- "Lecture" and "learn easier";

- "Lecture" and "Activity” and "Flexibility" (modest negative correlation);

• "Discussion" and "how to use";

- "Alone" and "Activity” and "Reflect” (negative correlation);

- “Groups” with “Creative” and “Activity” (negative correlation).

Orientation appears to demonstrate fit $Y$ by $X$ correlation with:

- "What learned" with Human Dynamics model of Mentally and Emotionally centered;

- "Why learned" with Human Dynamics model of Physically centered (negative correlation);

- "How to use" with Human Dynamics model of Emotionally centered.

"While learning" demonstrates a strong correlation with:

- "How to use" and "some distance from learning".

Pairwise correlation is demonstrated with:

- "Recall time" and "activity";

- "Groups" and "flexibility”;

- "Groups" and "recall time";

- "Spontaneity” and "creative";

- "Spontaneity" and "groups";

- "Structure” and "recall time” (negative correlation);

- "Structure” and "spontaneity”;

- "Structure" and "activity" (modest negative correlation);

- "Spontaneity and "flexibility” (modest correlation).

Dendrogram single linkage analysis identified "Structure" and "Reflection” with the highest coefficient 
distance for the facilitating factors, even though it demonstrated only a moderate similarity of approximately 72 . Regarding the aspect of what the reflection was focused on, the Dendrogram single linkage analysis identified the tightest linkage with "What was learned" and "How it integrates", followed by "How to use”, Learn easier", "Why learned" and "How learned". The first four linkages demonstrated similarity greater than 90.

Table 3 and Figure 5 provide information on the student' populations that were grouped by orientation and style of learning. Five groupings predominated for the classes surveyed, and the 79 students represent $83 \%$ of the total surveyed students. Although five grouping predominated, a total of 13 groupings were represented. (An analysis of student groupings utilizing Orientation, Style and Time-related reflective practices identified 32 combinations, however, the group with the greatest number of students contained 12 individuals (Task to be Accomplished, Discussion and Debate, Reflecting during learning), and the second largest group had 10 individuals (Tasks to be Accomplished, Discussion and Debate, Reflecting Some Later). The five groups are: (02 = Orientation element \#2, S1 = Style element \#1, etc., as noted for Table 3).

- Tasks to be Accomplished (O2) \& Reading \& Studying Alone (S1) (11 students);

- Tasks to be Accomplished (O2) \& Discussion and Debate with Classmates (S2) (29 students);

- Tasks to be Accomplished (O2) \& Group Work (S3) (23 students);

- Tasks to be Accomplished (O2) \& Lecture (S4) (10 students);

- Principles (O1) \& Discussion and Debate with Classmates (6 students).

For the composite of the five groups above, regarding the factors that were surveyed for these groups, the three factors that demonstrated the highest scorings were Creative (mean of 6.85, standard deviation of 1.54), How to Use (mean of 6.77, standard deviation of 2.07), and Flexible (mean of 6.69, standard deviation of 1.32). The three factors that demonstrated the lowest scorings were Structure (mean of 4.54, standard deviation of 1.83), Recall Time (mean of 4.86, standard deviation 1.56). and How Learned (mean of 5.00, standard deviation of 2.12). The three factors with the tightest distribution were Flexible (standard deviation of 1.32, mean of 6.69, Creative (standard deviation of 1.54, mean of 6.85), and Recall Time (standard deviation of 1.56, mean of 4.86). The three factors with the greatest deviation were Learn Easier (standard deviation of 2.34, mean of 5.77), How Integrates (standard deviation of 2.28, mean of 6.14), and Spontaneity (standard deviation of 2.20, mean of 5.51).

Regarding the five groups, Principles and Discussion generally scored the factors higher than the other groups (mean score of 6.22 with a standard deviation of 1.63), while Tasks to be Accomplished and Lecture tended to score the lowest of the five groups (mean score of 5.33 with a standard deviation of 2.11).

The highest and lowest factor scores for the different groups were:

- Tasks to be Accomplished (O2) \& Reading \& Studying Alone (S1) (How to Use (7.64), Structure (4.18));

- Tasks to be Accomplished (O2) \& Discussion and Debate with Classmates (S2) (Creative (7.41), How Learn (4.48));

- Tasks to be Accomplished (O2) \& Group Work (S3) (Groups (7.61), Structure (4.83));

- Tasks to be Accomplished (O2) \& Lecture (S4) (Flexible (6.9), How Learned (4.33));

- Principles (O1) \& Discussion and Debate with Classmates (S2) (How to Use (7.2), Structure (4.0)).

Questions (6) (Idea (1) vs. Activity (9)), (8) (quick recall (1), vs. long time lots of detail (9)), (10) (detailed info to start (1) vs. jump right in (9), (11) (Lot of structure (1), vs. no structure (9)), are thought to be representative of HD dynamics markers. 
For Tasks to be Accomplished (O2) \& Discussion and Debate with Classmates (S2):

- Many individuals (29) and scores were across the range for the various questions;

- Scores towards "idea” on \#6 tend to align with emotionally centered individuals. Scores towards "activity" on \#6 tend to align with physically centered individuals. Middle scores don’t appear to align with any particular dynamic.

Tasks to be Accomplished (O2) \& Group Work (S3):

- Twenty-three individuals and response similar to O2, S2;

- Tasks to be Accomplished (O2) \& Lecture (S4);

- Ten individuals and response similar to O2, S2, but somewhat less pronounced (more variation in response scores);

- Tasks to be Accomplished (O2) \& Reading \& Studying Alone (S1);

- Eleven individuals and high activity score tends to align with physically centered, but less clear for idea scores.

Principles (O1) \& Discussion and Debate with Classmates (S2):

- Six individuals and similar to above observations.

Contour Plot analysis suggests the following correlation. It should be noted that it is believed (from a combination of Orientation and Style rankings from the students) that individuals demonstrating characteristics of Mentally-Centered individuals (Human Dynamics model) are located near the bottom left of the contour plot, Emotionally-Centered individuals are located near the top to middle of the contour plot, and Physically-Centered individuals are located near the bottom right of the contour plot for Figure \#7 above).

- How to learn easier, how it integrates, how to use the knowledge, and what was learned are more strongly identified with Emotionally-Centered individuals, and only moderately identified with Physically-Centered individuals;

- Why it was learned, and how it was learned was minimally identified with any dynamic;

- Reflecting on learning was more strongly identified with Emotionally-Centered individuals;

- Reflecting on the learning at some time later was more strongly identified with Emotionally-Centered and Physically-Centered individuals;

- High Activity level, creativity, and self-starter were more closely identified with Emotionally-Centered individuals;

- Structure was important for Emotionally-Centered individuals;

- Group participation was important for Physically-Centered individuals.

\section{Discussion}

Several caveats might be noted at the beginning of this discussion. The study analyzed the results of 95 anonymous students' survey submissions, yet the number of students involved is somewhat small. It would be preferable to have more data to analyze, and it is anticipated that this will be accomplished in time. Most of the results from this analysis are tentative in nature due to sample size, but it is possible that in utilizing a Likert scale of 9 (with an attempt to improve resolution) may have contributed to less clarity with the data than desired. Caution might also be expressed with the lack of precision with the word choices used in the questions asked 
within the survey. This caution is further amplified by the recognition (within the Human Dynamics model) that individual words have specific nuances of meaning for the different dynamics. A further challenge that must be noted is that the attempt to identify a particular dynamic with a set of responses from the survey is very difficult, because none of the students who participated in the survey had any exposure with the Human Dynamics model, and their responses to the various questions are in a manner that is consistent with their current view of the world. The reason that this is a particular challenge, is that to-date, all more than 100,000 individuals who have been exposed to the Human Dynamics model have self-identified their dynamic after participating in a work shop that normally is conducted over a two- or four- day period of time. No instrument has been designed that helps an individual identify their particular dynamic external of a workshop. Consequently, there is a certain level of presumption that is inherent in this study, because it has been conjectured that it might be possible to identify different dynamics by their specific response to select questions.

It is suggested that from the survey, questions \#5 (idea oriented or task oriented), \#7 (time needed to remember and detail level), \#9 (detailed instructions, or ability to work with little direction), and \#10 (great deal of structure, or essentially no structure needed) might be considered as "marker" questions to help identify a particular dynamic. In this regard, and in alignment with findings from the Human Dynamic work, individuals who tend to be more idea oriented, need little direction to begin a task, remember things quickly, and need little structure tend to be of the emotional dynamics (Emotional Mental and Emotional Physical). Individuals that tend to be more task related, need significant direction before beginning a task, require significant amount of time to remember things but remember in great detail, and require a significant amount of structure, tend to be of the Physical dynamics (Physical Mental, and Physical Emotional).

The results of the study suggest that different reflective practices, and the focus of those reflective practices, exist across the student population. As such, it appears that individuals may inherently tend towards either a “thinking-in-action" or "thinking-on-action" strategy for reflection (as proposed by Schon), and this study preliminarily suggests that Emotionally-Centered individuals demonstrate a greater tendency toward "thinking-in-action", while Physically-Centered individuals demonstrate a greater tendency towards "thinking-on-action”. Further, Figure 7 (and other Contour Plots) suggests a variety of reflective practices and focus for the different dynamics. While Figure 7 does not negate Gibbs' position, the inclusion of emotion elements in reflection may not be universally required (Gibbs, 1988).

Table 1 proposes that different learning styles may exist not only for individuals, but also for the composite of the class. Therefore, it is likely that instructors should be cognizant of the general make-up of class participants in order to structure an instructional delivery methodology that would be most appropriate to the composite class. This is not to advocate that instructors ignore the needs of the individual student, but it is recommended that being aware of group dynamics may help to facilitate a more productive learning environment for the composite members of the group.

Table 1 also suggests that for the preferred learning style of "reading and studying alone”, 37.5\% of the grad students used this style of learning. This appears logical from the perspective that these students were all distance-learning students, and by default have to read and study alone. What is not clear, is whether this is truly their preferred learning process, or whether it is a default learning process due to the nature of how the class was conducted. This observation is also true for the $10 \%$ of the undergraduate night students. It is not clear if this is 
their preferred learning style, or a default due to generally working full time during the day, and needing to take classes at night in order to complete their education.

According to the Human Dynamics model, the orientation of "task to be completed" should be the predominant orientation for Physically centered individuals. However, the large number of students noting this as their preferred orientation seems to be larger than expected for the make-up of the student population. The high proportion of students with "tasks to be completed" orientation may be an artifact of societal and school pressures. This may also be true for the graduate students within the study, but it is also possible that since these students were all full-time working adults, and an expressed motivation for taking graduate courses was to enhance their careers, the orientation of "tasks to be completed" may be directly related to their short-term goal of completing the program in conjunction with the factor reflecting on "how to use" the material that was being learned.

With regard to what is being reflected on by students, the study indicates that students reflect primarily on "How to Use it" and "What is Learned" (in that order). Little reflection is given to "How it was Learned", or "How to Learn Easier". Only modest reflection effort was recorded for "Why it was Learned", and "How it Integrates with other Learning". Considering the "task" orientation of the students in the study, it is easy to understand why reflection for "What is Learned" and "How to Use it" predominate in their thinking. These reflective practices will likely enhance the student's ability to actualize the course materials into productive output in the student's professional lives. Encouraging reflection on how the material "Integrates with other Learning”, and "Why it Was Learned” may help the student develop a greater appreciation for the complexity of the world and help to acquire a greater appreciation for a total system's perspective of the world. Regarding the two questions of "How it was Learned" and "How to Learn Easier", considering that the world is rapidly transitioning to a knowledge-based economy, and that life-long learning will be a necessity for individual prosperity, encouraging reflection on these questions may help students become more proficient at continuous learning.

It is interesting that a significant number of students do not reflect (or reported that they do not reflect), and that an even larger number of students do not reflect in a consistent manner. Therefore the results of Figure 1 suggest that a significant number of students should be encouraged to expand their reflective practices. The reason for suggesting this action is to help improve the student's learning and comprehension of the material being studied (in agreement with Pyc and Rawson), and to help develop an ongoing practice of reflective thinking that might be of use in their professional lives. A potential strategy that might be useful is to require weekly reflective pieces from each student. A simple technique that might be useful is a slight modification of what Rolfe has been proposed (Rolfe, 2001) where the students would write responses to four questions: "What", "For What", "So What", and "Now What". A different strategy may be to have the students briefly respond to the reflection questions posed in this study ("What was Learned”, "Why it was Learned”, etc.). To encourage both "reflection-in-action" and "reflection-on-action", it is proposed that the instructor utilize two complementary strategies for student reflection: requiring brief written papers immediately after the significant learning, and a "summary" reflective paper at the end of the course.

\section{Conclusion}

It is believed that the variety of learning preference for the students (see Table 1) noted in this study is not 
significantly different for most well integrated classes. This poses a challenge for the instructor in being able to effectively present material so that each student is able to master the requirements of the course. However, being aware of the variety of learning preferences allows the instructor to design his/her course in a manner that addresses each of the learning preferences. Therefore, although it may not be possible to completely satisfy each student for every element of the course design, at least each learning preference will be available to the student body. Further, it is suggested that to enhance the utilization of reflective practices, the instructor establishes a structure in his/her class that mandates reflection immediately after a particular learning event providing structure aligns with the results of the analysis in this study.

From a global perspective, there appears to be general alignment with this study and the Human Dynamics model for individuals who scored the various questions at the extreme ranges in the Likert scale (both high and low). However, correlation with the Human Dynamics model becomes "murkier" as the scores tend toward the middle of the scale, and this may be potentially attributed to the range of responses that individuals will demonstrate within any particular dynamic (as noted above), and it may also suggest that typical quantitative statistical analysis does not fully address the complexity of the human population, and that diagnostic tools similar to Contour Plotting, even though requiring greater subjectivity for interpretation, may be more useful.

Useful information resulted from this study. However, this study generates many questions. While it is recognized that this study is imperfect due to the caveats noted at the beginning of the "Discussion" section of this paper, it is possible that with further study greater correlation and understanding might be achieved.

\section{References}

Argyris, C. (1982). Reasoning, learning, and action. San Francisco, C.A.: Jossey-Bass.

Bergstrom, B. (2004). Every child has specific needs. Stockholm, Sweden: Runa Forlung.

Boud, D., Cohen, R., \& Walker, D. (1993). Using experience for learning. Buckingham, U.K.: Society for Research into Higher Education and Open University Press.

D’Aveni, R. A. (1994). Hyper-competition: Managing the dynamics of strategic maneuvering. New York, N.Y.: Free Press.

Drucker, P. (1967). The effective executive. Oxford, U.K.: Butterworth-Heinemann.

Gibbs, G. (1988). Learning by doing: A guide to teaching and learning methods. London, U.K.: Oxford Centre fro Staff and Learning Development, Oxford Polytechnic.

King, P., \& Kitchener, K. (1981). Developing reflective judgment. San Francisco, C.A.: Jossey-Bass.

Magolda, B. (2001). Making their own way: Narratives for transforming higher education to promote self-development. Sterling, V.A.: Stylus Publishing.

McDrury, J., \& Alterio, M. (2002). Learning through storytelling in higher education using reflection and experience to improve learning. London, U.K.: The Higher Education Academy. Kogan Page.

Pyc, M., \& Rawson, K. (2010, November 6). How testing improves memory. Science News, 178(10), 16.

Rolfe, G., Freshwater, D., \& Jasper, M. (2001). Critical reflection for nursing and the helping professions. Basingstoke, U.K.: Palgrave.

Schon, D. (1983). The reflective practitioner: How professionals think in action. Basic Books.

Seagal, S., \& Horne, D. (1997). Human dynamics: A new framework for understanding people and realizing the potential in our organizations. Cambridge, M.A.: Pegasus Communications.

Sterman, J. D. (2000). Business dynamics: Systems thinking and modeling for a complex world. Boston, M.A.: Irwin McGraw-Hill. 